\title{
Mastery of Information Technology among Malay Language Students
}

\section{Zuraini Jusoh}

To Link this Article: http://dx.doi.org/10.6007/IJARSS/v10-i11/7906

DOI:10.6007/IJARSS/v10-i11/7906

Received: 27 Septemer 2020, Revised: 25 October 2020, Accepted: 15 Novemer 2020

Pulished Online: 29 Novemer 2020

In-Text Citation: (Jusoh, 2020)

To Cite this Article: Jusoh, Z. (2020). Mastery of Information Technology among Malay Language Students. International Journal of Academic Research in Business and Social Sciences. 10(11), 983-995.

\section{Copyright: (c) 2020 The Author(s)}

Pulished y Human Resource Management Academic Research Society (www.hrmars.com)

This article is pulished under the Creative Commons Attriution ( $C C$ Y 4.0 ) license. Anyone may reproduce, distriute, translate and create derivative works of this article (for oth commercial and non-commercial purposes), suject to full attriution to the original pulication and authors. The full terms of this license may e seen at: http://creativecommons.org/licences/v/4.0/legalcode

\section{Vol. 10, No. 11, 2020, Pg. 983 - 995}

Full Terms \& Conditions of access and use can e found at http://hrmars.com/index.php/pages/detail/pulication-ethics 


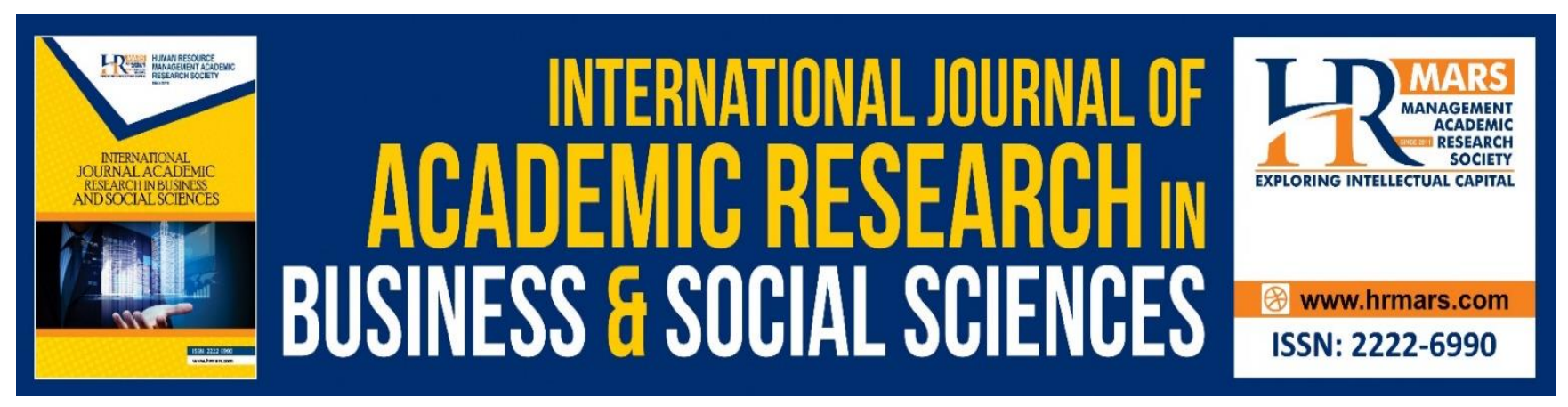

\title{
Mastery of Information Technology among Malay Language Students
}

\author{
Zuraini Jusoh (PhD) \\ Malay Language Department, Faculty of Modern Language and Communication, \\ Universiti Putra Malaysia \\ Email: zurainijusoh@upm.edu.my
}

\begin{abstract}
Astract
Information and communication technology skills are some of the most important components that are emphasized y employers today. This rapid technological development makes the curriculum in institutions of higher learning to apply technological elements in teaching and learning. Students are not left ehind in the field of language to enale them to compete in the career market after completing their studies. To that end, a survey using a questionnaire was conducted to identify the level of information and communication technology skills among students in the field of language. A total of 30 samples from $9(30.0 \%)$ men and $21(70.0 \%)$ were female students in the fifth semester to the final semester of the achelor of Arts (Malay Language and Linguistics). In total, 11(36.7\%) students are moderately skilled and $19(63.3 \%)$ are highly skilled in information and communication technology skills. Most of the students surveyed also spend more than seven hours surfing the Internet which is usually rowsed through their respective smartphones. Usually, these students surf the Internet to access email, for entertainment and curiosity.
\end{abstract}

Keywords: Language Learners, Technological Skills, Career Opportunities, Higher Education, Malay Language

\section{Introduction}

The explosion of gloalization demands Malaysia, a country heading towards a developed country to compete in various fields. This competition is included in the world of education to produce world-class education. If we look at the rate of development of national roadand penetration, it is noticeale that the development occurred drastically, from $24.8 \%$ in 2009 to $55.6 \%$ in 2011 (Mohamed et al., 2012). This is ecause information and communication technology ecomes the fastest growing technology after the computer and communication revolution (Omar et al., 2006). The importance of the field of information and communication technology is further evidenced y the launch of the ASEAN Communication and Information Technology Master Plan 2015 on 14 January 2014 and the estalishment of the Multimedia Super Corridor which is one of the strategic steps to achieve developing country status y 2020 (Alias, 2004). To get through the current gloalization, the digital age is considered very important and a priority of developing countries (Mohamed et al., 2012). The rapid development of technology has prompted employers around the world to place information 
technology skills as one of the conditions and criteria of employees required y a company (Jusoh, 2018).

This need has ecome an important agenda of the country and is the responsiility of higher education to produce a highly competitive workforce and meet the needs of the market (Madar et al., 2008). The same goes for local institutions of higher learning as most of them have e-learning policies that mandate the use of e-learning among lecturers and students (Emi, 2010). Regardless of the field of study, knowledge of information technology is incorporated into teaching and learning activities. This is proved $y$ the existence of the Language in Multimedia courses to e taken y students, like the achelor of Arts Malay Language and Linguistics, Faculty of Modern Language and Communication, Universiti Putra Malaysia. The importance of this technology is reflected when the 2017 curriculum review has changed the name of this course to Multimedia in Language.

The need for this technological development also hit Malaysia when the development rate of roadand coverage in the country increased drastically in a short time. The Ministry of Communications and Multimedia Malaysia and the cyer law act in Malaysia have also een created as a result of the rapid development of this technology. Apart from that, the former Prime Minister also launched the ASEAN Communication and Information Technology Master Plan 2015 on 14 January 2014 which proves that the country is not left ehind in pursuing the rapid development of information and communication technology. This is ecause the Internet is a gloal media that is powerful and has the potential to control the whole world (Pitchan et al., 2017).

The Internet plays an important role in the world of communication and information and is used as a medium to convey and otain information (Pitchan et al., 2017). This is detailed y Mustafa and Hamzah (2011) who said that users usually use the Internet for various purposes such as finding friends, having fun, and getting support or help from the online community from time to time. This situation makes society more literate to information technology regardless of the generation they were orn in. This can e oserved as not only every group of people owns a smartphone ut also has its internet network offered y telecommunication companies. Therefore, it is not impossile when employers place these information technology skills as a prerequisite for employee selection. This is ecause computer skills are among the generic skills that are important and need to e mastered y students in preparation to enter the world of work (Mohd et al., 2001). Davis (1997) reported that $83.3 \%$ of employers have high expectations of graduates in computer skills and it is one of the factors seen for hiring. Among the computer literacy skills are the aility to use certain software applications for ovious tasks such as word processing, email, and the internet (ers, 2010). The development of science and technology also has a positive influence on learning (Auzar, 2012). This opinion is supported y Aladdin et al. (2004) who said that the use of computers has een proven to have considerale and dynamic potential in language teaching and learning. This is ecause multimedia application software helps the teaching process of teachers to e more flexile and effective (Mahamod \& Mohamad, 2011).

However, the analysis of the gap of marketaility characteristics according to the priorities conducted y Ismail (2012) especially for the needs of information and communication technology skills, found that the actual performance of graduates is still elow the expected performance of employers. This should not have happened ecause these graduates were orn in an era of rapid technological development. According to Agus et al. (2016) individuals orn in the early 2000s, are known as Generation Z or Generation Net. However, Suthagar et al. (2011) argued that this generation was orn after 1985. This generation is said to e orn during 
the rapid development of technology makes them sensitive to information and communication technology (Agus et al., 2016). The Net Generation or Millennium Generation is said to have the skills and know how to use various types of digital technology (Kiam-Sam \& Adul, 2014). However, information technology skills and knowledge still need to e given attention (Suthagar et al., 2011). This opinion is supported y Olingers (2005) who states that although this generation can use a variety of technology applications without manual instruction, understanding the technology or quality of its resources may e difficult. Therefore, this survey was conducted to identify to which extent students' skills are categorized in this Net Generation in information and communication technology especially skills in using asic computer software.

\section{Methods}

This survey study was conducted on students who took the M3410 Multimedia in Language course in the first semester of 2017/2018 at the Faculty of Modern Languages and Communication, Universiti Putra Malaysia. This course is compulsory for achelor of Arts Malay Language and Linguistics students in the second year of their studies. A total of 30 samples answered this questionnaire, namely $9(30 \%)$ were male students and $21(70 \%)$ were female students. Looking at the distriution of the nation, the majority of study participants are Malays, which is 25 people (83.3\%). Participants in this study met the characteristics of the Net Generation as they were orn etween the year 1992 to 1995, which is after 1985 . This survey was conducted to achieve the following ojective:

1. To identify the level of information and communication technology skills among students in the field of language

This questionnaire was distriuted in the first week of the lecture to enale lecturers to get an initial overview of information technology mastery among students. The next step is to help the lecturer formulate a teaching and learning plan appropriate to the student's mastery. A total of 118 items were uilt which were divided into five parts, namely measuring the mastery of information technology, word processing software (Word), presentation software (PowerPoint), electronic spreadsheets (Excel), and mastery of skills using the Internet. Data were otained through questionnaires using ordinal scales of semantic differences and analyzed using IM SPSS 22 (Statistical Package for the Social Science) software. This constructed item as a whole otained a high reliaility value when the Cronach Alpha coefficient value, $\alpha=0.987$ was recorded. Whereas, the Cronach Alpha coefficient for each part or construct etween, $\alpha=0.933$ to $\alpha=0.976$ indicates that this constructed item has high reliaility. According to Guilford (1956), $\alpha=0.7$ is most satisfactory and Pallant (2001) suggests that the alpha index value, $\alpha=0.7$ and aove is good for items ten and aove.

\section{Results}

Discussion of the findings of this study is made ased on three levels of mastery, namely unskilled with a mean score value etween 1.00 to 4.00, moderately skilled with a mean score etween 4.01 to 7.00 , and skilled with a mean score etween 7.01 to 10.00 . ased on Tale 1 , students were found to e very skilled in information technology skills ( $M=7.34 ; S P=1.28$ ). However, they were found to e moderately skilled in asic computer skills ( $M=6.57 ; S P=1.32$ ) and electronic spreadsheets (Excel) $(M=5.97 ; \mathrm{SP}=2.11$ ) which are the asic software that they often use throughout their higher education. There is $1(3.3 \%)$ who is not proficient in asic 
computer software and presentation software (PowerPoint) and a total of $7(23.3 \%)$ students are not proficient in electronic spreadsheet software (Excel). On the other hand, students were found to e proficient in using the Internet when they recorded the highest mean value, which is 8.03 with a standard deviation value of 1.24 .

Tale 1. Level of Information Technology Skills

\begin{tabular}{|c|c|c|c|c|c|}
\hline \multirow[t]{2}{*}{ Software Skills } & \multicolumn{3}{|c|}{ Mastery Level } & \multirow[b]{2}{*}{ Mean } & \multirow[b]{2}{*}{ SD } \\
\hline & Unskilled & $\begin{array}{l}\text { Moderately } \\
\text { Skilled }\end{array}$ & Skilled & & \\
\hline $\begin{array}{l}\text { Computer } \\
\text { asics }\end{array}$ & $1(3.3 \%)$ & $18(60.0 \%)$ & $11(36.7 \%)$ & 6.57 & 1.32 \\
\hline $\begin{array}{l}\text { Word } \\
\text { Processing }\end{array}$ & 0 & $5(16.7 \%)$ & $25(83.3 \%)$ & 7.89 & 1.30 \\
\hline PowerPoint & $1(3.3 \%)$ & $9(30.0 \%)$ & $20(66.7 \%)$ & 7.66 & 1.60 \\
\hline Excel & $7(23.3 \%)$ & $14(46.7 \%)$ & $9(30.0 \%)$ & 5.97 & 2.11 \\
\hline Internet & 0 & $7(23.3 \%)$ & $23(76.7 \%)$ & 8.03 & 1.24 \\
\hline Total & 0 & $11(36.7 \%)$ & $19(63.3 \%)$ & 7.34 & 1.28 \\
\hline
\end{tabular}

Although students were found to have mastered these information technology skills, the overall mean value is $11(36.7 \%)$ out of 30 students for moderately proficient. This figure is quite large and should not happen among Net Generation students and students in institutions of higher learning ecause not only they were orn in the rapidly evolving technology era ut are provided with various technological facilities in higher education under current demands.

No. Items

\begin{tabular}{llll}
\multicolumn{2}{l}{ Mastery Level } & \\
\hline Unskilled & $\begin{array}{l}\text { Moderately } \\
\text { Skilled }\end{array}$ & Skilled Mean SD
\end{tabular}




\begin{tabular}{|c|c|c|c|c|c|c|}
\hline 1. & Word & 0 & $4(13.3 \%)$ & $26(86.7 \%)$ & 8.47 & 1.28 \\
\hline 2. & Flash & $18(60.0 \%)$ & $10(33.3 \%)$ & $2(6.7 \%)$ & 3.60 & 2.24 \\
\hline 3. & Paint & $4(13.3 \%)$ & $12(40.0 \%)$ & $14(46.7 \%)$ & 6.67 & 2.09 \\
\hline 4. & Excel & $5(16.7 \%)$ & $18(60.0 \%)$ & $7(23.3 \%)$ & 5.93 & 1.84 \\
\hline 5. & Email & $1(3.3 \%)$ & $3(10.0 \%)$ & $26(86.7 \%)$ & 8.73 & 1.46 \\
\hline 6. & Graphics & $9(30.0 \%)$ & $16(53.3 \%)$ & $5(16.7 \%)$ & 5.53 & 2.26 \\
\hline 7. & Internet & $1(3.3 \%)$ & $5(16.7 \%)$ & $24(80.0 \%)$ & 8.57 & 1.55 \\
\hline 8. & Antivirus & $7(23.3 \%)$ & $13(43.3 \%)$ & $10(33.3 \%)$ & 6.40 & 1.90 \\
\hline 9. & Calculator & $4(13.3 \%)$ & $12(40.0 \%)$ & $14(46.7 \%)$ & 7.33 & 2.22 \\
\hline 10. & Photoshop & $17(56.7 \%)$ & $10(33.3 \%)$ & $3(10.0 \%)$ & 4.00 & 2.23 \\
\hline 11. & File Format & $11(36.7 \%)$ & $14(46.7 \%)$ & $5(16.7 \%)$ & 5.03 & 2.51 \\
\hline 12. & PowerPoint & $2(6.7 \%)$ & $6(20.0 \%)$ & $22(73.3 \%)$ & 8.07 & 1.70 \\
\hline 13. & Hardware & $9(30.0 \%)$ & $13(43.3 \%)$ & $8(26.7 \%)$ & 5.90 & 2.32 \\
\hline 14. & Print Screen & $4(13.3 \%)$ & $6(20.0 \%)$ & $20(66.7 \%)$ & 7.83 & 2.07 \\
\hline 15. & Sticky Notes & $3(10.0 \%)$ & $8(26.7 \%)$ & 19(63.3\%) & 7.73 & 2.23 \\
\hline 16. & Snipping Tool & $6(20.0 \%)$ & $10(33.3 \%)$ & $14(46.7 \%)$ & 6.53 & 2.80 \\
\hline 17. & Windows/Office & $1(3.3 \%)$ & $10(33.3 \%)$ & $19(63.3 \%)$ & 7.40 & 2.01 \\
\hline 18. & ackup Program & $8(26.7 \%)$ & $14(46.7 \%)$ & $8(26.7 \%)$ & 5.60 & 2.37 \\
\hline 19 & Restore Program & $7(23.3 \%)$ & $14(46.7 \%)$ & $9(30.0 \%)$ & 5.83 & 2.38 \\
\hline 20. & Hardware Function & $8(26.7 \%)$ & $12(40.0 \%)$ & $10(33.3 \%)$ & 5.87 & 2.54 \\
\hline 21. & Upload document & $1(3.3 \%)$ & $7(23.3 \%)$ & $22(73.3 \%)$ & 8.07 & 1.57 \\
\hline 22. & Download document & $2(6.7 \%)$ & $6(20.0 \%)$ & $22(73.3 \%)$ & 8.13 & 1.66 \\
\hline 23. & Fax \& scanner machine & $4(13.3 \%)$ & $12(40.0 \%)$ & $14(46.7 \%)$ & 6.73 & 2.13 \\
\hline 24. & $\begin{array}{l}\text { Data analyzing using } \\
\text { IM SPSS }\end{array}$ & $17(56.7 \%)$ & $12(40.0 \%)$ & $1(3.3 \%)$ & 3.80 & 2.23 \\
\hline & & $1(3.3 \%)$ & $18(60.0 \%)$ & $11(36.7 \%)$ & 6.57 & 1.32 \\
\hline
\end{tabular}

A total of 24 items was constructed to measure the level of asic computer skills among the students of Malay language and linguistics at the Faculty of Modern Language and Communication, Universiti Putra Malaysia. Out of 24 items, students were found to e unskilled in 3(12.5\%) items, namely Flash ( $\mathrm{M}=3.60$; $\mathrm{SP}=2.24)$, Photoshop $(\mathrm{M}=4.00$; $\mathrm{SP}=$ $2.23)$, and IM SPSS ( $M=3.80 ; S P=2.23)$. Students were found to e moderately proficient in $11(45.8 \%)$ items as they otain a mean value etween 5.03 to 6.73 and proficient in 10 items $(41.7 \%)$ as they otain a mean value etween 7.33 to 8.73 . Students were found to e proficient in using email when they recorded the highest mean value, which is 8.73 with a standard deviation value of 1.46 .

Overall, the students who were the sample of this study were moderately proficient in mastering asic computer skills. This matter needs to e emphasized ecause as students in institutions of higher learning and in a research university, the students should master these asic skills. Although courses related to information and multimedia technology as well as the asorption of technological elements throughout the study has een availale, they were found to have not mastered them properly. This should e taken seriously as only one out of 24 items in this section has no unskilled students. On the other hand, at least one of 18 people is not proficient in the remaining 23 items.

TALE 3. Level Word Processing Skills

No. Items

Mastery Level 


\begin{tabular}{|c|c|c|c|c|c|c|}
\hline & & Unskilled & $\begin{array}{l}\text { Moderate } \\
\text { ly Skilled }\end{array}$ & Skilled & Mean & SD \\
\hline 1. & Picture & 0 & $8(26.7 \%)$ & $22(73.3 \%)$ & 8.27 & 1.34 \\
\hline 2. & Shapes & 0 & $11(36.7 \%)$ & $19(63.3 \%)$ & 8.20 & 1.47 \\
\hline 3. & ullets & 0 & $7(23.3 \%)$ & $23(76.7 \%)$ & 8.33 & 1.24 \\
\hline 4. & Symol & $1(3.3 \%)$ & $7(23.3 \%)$ & $22(73.3 \%)$ & 8.17 & 1.39 \\
\hline 5. & Margins & $2(6.7 \%)$ & $6(20.0 \%)$ & $22(73.3 \%)$ & 7.93 & 1.91 \\
\hline 6. & Equation & $4(13.3 \%)$ & $15(50.0 \%)$ & $11(36.7 \%)$ & 6.63 & 2.19 \\
\hline 7. & Text ox & 0 & $7(23.3 \%)$ & $23(76.7 \%)$ & 8.43 & 1.48 \\
\hline 8. & Font Size & 0 & $2(6.7 \%)$ & $28(93.3 \%)$ & 8.90 & 1.16 \\
\hline 9. & Find Word & $1(3.3 \%)$ & $8(26.7 \%)$ & $21(70.0 \%)$ & 8.03 & 1.73 \\
\hline 10. & Orientation & $3(10.0 \%)$ & 10(33.3\%) & $17(56.7 \%)$ & 7.23 & 2.28 \\
\hline 11. & Page Color & $4(13.3 \%)$ & $8(26.7 \%)$ & $18(60.0 \%)$ & 7.50 & 2.18 \\
\hline 12. & Watermark & $4(13.3 \%)$ & $13(43.3 \%)$ & $13(43.3 \%)$ & 6.90 & 2.06 \\
\hline 13. & Paper Size & $1(3.3 \%)$ & $8(26.7 \%)$ & $21(70.0 \%)$ & 7.83 & 1.62 \\
\hline 14. & Numering & $1(3.3 \%)$ & $6(20.0 \%)$ & $23(76.7 \%)$ & 8.33 & 1.42 \\
\hline 15. & Draw Tale & $1(3.3 \%)$ & $4(13.3 \%)$ & $25(83.3 \%)$ & 8.40 & 1.38 \\
\hline 16. & Page reak & $2(6.7 \%)$ & $8(26.7 \%)$ & $20(66.7 \%)$ & 7.83 & 1.86 \\
\hline 17. & Merge Cells & $1(3.3 \%)$ & $8(26.7 \%)$ & $21(70.0 \%)$ & 7.70 & 1.97 \\
\hline 18. & Word Count & $2(6.7 \%)$ & $7(23.3 \%)$ & $21(70.0 \%)$ & 7.80 & 1.71 \\
\hline 19 & Insert Tale & $1(3.3 \%)$ & $4(13.3 \%)$ & $25(83.3 \%)$ & 8.50 & 1.41 \\
\hline 20. & Delete Tale & 0 & $4(13.3 \%)$ & $26(86.7 \%)$ & 8.70 & 1.12 \\
\hline 21. & Page orders & $2(6.7 \%)$ & $7(23.3 \%)$ & $21(70.0 \%)$ & 7.90 & 1.81 \\
\hline 22. & Page Numer & 0 & $3(10.0 \%)$ & $27(90.0 \%)$ & 8.70 & 1.12 \\
\hline 23. & Text Direction & $2(6.7 \%)$ & $13(43.3 \%)$ & $15(50.0 \%)$ & 7.30 & 2.07 \\
\hline 24. & Replace Word & $2(6.7 \%)$ & $9(30.0 \%)$ & $19(63.3 \%)$ & 7.93 & 1.66 \\
\hline 25. & Track Changes & $7(23.3 \%)$ & $11(36.7 \%)$ & $12(40.0 \%)$ & 6.27 & 2.57 \\
\hline 26. & Change the font & $1(3.3 \%)$ & $5(16.7 \%)$ & $24(80.0 \%)$ & 8.33 & 1.67 \\
\hline 27. & Paragraph Marks & $5(16.7 \%)$ & $10(33.3 \%)$ & $15(50.0 \%)$ & 6.77 & 2.67 \\
\hline 28. & Header \& Footer & $1(3.3 \%)$ & $6(20.0 \%)$ & $23(76.7 \%)$ & 8.10 & 1.63 \\
\hline 29. & Insert Row & $1(3.3 \%)$ & $6(20.0 \%)$ & $23(76.7 \%)$ & 8.37 & 1.35 \\
\hline 30. & Text Highlight Color & 0 & $7(23.3 \%)$ & $23(76.7 \%)$ & 8.33 & 1.37 \\
\hline 31. & Insert Columns & $1(3.3 \%)$ & $5(16.7 \%)$ & $24(80.0 \%)$ & 8.50 & 1.38 \\
\hline 32. & oarders \& Shading & $3(10.0 \%)$ & $8(26.7 \%)$ & $19(63.3 \%)$ & 7.60 & 2.01 \\
\hline 33. & $\begin{array}{ll}\text { Set } & \text { Proofing } \\
\text { Language } & \\
\end{array}$ & $6(20.0 \%)$ & $12(40.0 \%)$ & $12(40.0 \%)$ & 6.67 & 2.26 \\
\hline Total & & 0 & $5(16.7 \%)$ & $25(83.3 \%)$ & 7.89 & 1.30 \\
\hline
\end{tabular}

Tale 3 shows the level of mastery of skills in using word processing software among students. Students were found to e proficient in using this software when $28(84.8 \%)$ items they mastered and 5(15.2\%) items were moderately proficient. However, if we look at all 33 items, $25(75.8 \%)$ items recorded etween $1(3.3 \%)$ to $7(23.3 \%)$ students who are not proficient in these skills, especially in Track Changes ( $M=6.27$; $S P=2.57)$. On the other hand, only 8 items (Picture, Shape, ullets, Text Size, Font Size, Delete Tale, Page Numer and Text Highlight Color) did not have the numer of unskilled students and students were found to e the most skilled in Font Size ( $\mathrm{M}=8.90 ; \mathrm{SP}=1.16)$. 
TALE 4. Level of PowerPoint Software Skills

\begin{tabular}{|c|c|c|c|c|c|c|}
\hline \multirow[t]{2}{*}{ No. } & \multirow[t]{2}{*}{ Items } & \multicolumn{3}{|c|}{ Mastery Level } & \multirow[b]{2}{*}{ Mean } & \multirow[b]{2}{*}{ SD } \\
\hline & & Unskilled & $\begin{array}{l}\text { Moderately } \\
\text { Skilled }\end{array}$ & Skilled & & \\
\hline 1. & Crop & $1(3.3 \%)$ & $4(13.3 \%)$ & $25(83.3 \%)$ & 8.60 & 1.48 \\
\hline 2. & Rotate & $1(3.3 \%)$ & $5(16.7 \%)$ & $24(80.0 \%)$ & 8.50 & 1.50 \\
\hline 3. & Hyperlink & $4(13.3 \%)$ & $13(43.3 \%)$ & $13(43.3 \%)$ & 6.87 & 2.30 \\
\hline 4. & Print Slide & 0 & $4(13.3 \%)$ & $26(86.7 \%)$ & 8.90 & 1.09 \\
\hline 5. & Animation & $4(13.3 \%)$ & $11(36.7 \%)$ & $15(50.0 \%)$ & 7.03 & 2.36 \\
\hline 6. & Insert Video & $2(6.7 \%)$ & $10(33.3 \%)$ & $18(60.0 \%)$ & 7.67 & 1.97 \\
\hline 7. & Insert Audio & $2(6.7 \%)$ & $11(36.7 \%)$ & $17(56.7 \%)$ & 7.70 & 1.86 \\
\hline 8. & Picture Style & $1(3.3 \%)$ & $10(33.3 \%)$ & $19(63.3 \%)$ & 7.93 & 1.70 \\
\hline 9. & Insert Picture & 0 & $7(23.3 \%)$ & $23(76.7 \%)$ & 8.47 & 1.36 \\
\hline 10. & Insert Clip Art & 0 & $8(26.7 \%)$ & $22(73.3 \%)$ & 8.30 & 1.29 \\
\hline 11. & Group Ojects & $5(16.7 \%)$ & $12(40.0 \%)$ & $13(43.3 \%)$ & 6.77 & 2.54 \\
\hline 12. & Picture Effects & $2(6.7 \%)$ & $9(30.0 \%)$ & $19(63.3 \%)$ & 7.63 & 2.09 \\
\hline 13. & Artistic Effects & $4(13.3 \%)$ & $13(43.3 \%)$ & $13(43.3 \%)$ & 6.87 & 2.39 \\
\hline 14. & Change Picture & $1(3.3 \%)$ & $9(30.0 \%)$ & $20(66.7 \%)$ & 8.00 & 1.58 \\
\hline 15. & Change Theme & 0 & $9(30.0 \%)$ & $21(70.0 \%)$ & 8.13 & 1.48 \\
\hline 16. & Slide Orientation & $3(10.0 \%)$ & $8(26.7 \%)$ & $19(63.3 \%)$ & 7.73 & 2.20 \\
\hline 17. & Arrange Ojects & $5(16.7 \%)$ & $11(36.7 \%)$ & $14(46.7 \%)$ & 6.97 & 2.61 \\
\hline 18. & Rehearse Timing & $5(16.7 \%)$ & $11(36.7 \%)$ & $14(46.7 \%)$ & 6.90 & 2.45 \\
\hline 19 & ackground Style & $1(3.3 \%)$ & $10(33.3 \%)$ & $19(63.3 \%)$ & 7.63 & 1.90 \\
\hline 20. & Compress Picture & $5(16.7 \%)$ & $10(33.3 \%)$ & $15(50.0 \%)$ & 7.07 & 2.45 \\
\hline 21. & Set Up Slide Show & $2(6.7 \%)$ & $8(26.7 \%)$ & $20(66.7 \%)$ & 7.73 & 2.21 \\
\hline 22. & Insert Photo Alum & $2(6.7 \%)$ & $10(33.3 \%)$ & $18(60.0 \%)$ & 7.73 & 1.96 \\
\hline 23. & Remove ackground & $1(3.3 \%)$ & $9(30.0 \%)$ & $20(66.7 \%)$ & 7.87 & 1.96 \\
\hline 24. & $\begin{array}{l}\text { Change } \quad \text { Picture } \\
\text { order }\end{array}$ & $3(10.0 \%)$ & $8(26.7 \%)$ & $19(63.3 \%)$ & 7.67 & 2.15 \\
\hline 25. & $\begin{array}{l}\text { Picture Correction } \\
\text { Option }\end{array}$ & $4(13.3 \%)$ & $11(36.7 \%)$ & $15(50.0 \%)$ & 6.90 & 2.16 \\
\hline Total & & $1(3.3 \%)$ & $9(30.0 \%)$ & $20(66.7 \%)$ & 7.66 & 1.60 \\
\hline
\end{tabular}

To measure the level of mastery of presentation software or PowerPoint skills, a total of 25 items have een developed. Out of 25 items, 6(24.0\%) items were moderately skilled and the rest were proficient. Overall, students were found to e proficient in using this presentation software ( $M=7.66$; $S P=1.60)$. However, if we look at each item, we found that only four items (Print Slide, Insert Picture, Insert Clip Art and Change Theme) have no unskilled students. In contrast, $19(76.0 \%)$ items found that at least $1(3.3 \%)$ to $5(16.7 \%)$ did not master the skill.

TALE 5. Level of Excel Skills

\begin{tabular}{|c|c|c|c|c|c|c|}
\hline \multirow{2}{*}{ No. } & \multirow[t]{2}{*}{ Items } & \multicolumn{3}{|c|}{ Mastery Level } & \multirow[b]{2}{*}{ Mean } & \multirow[b]{2}{*}{ SD } \\
\hline & & Unskilled & $\begin{array}{l}\text { Moderately } \\
\text { Skilled }\end{array}$ & Skilled & & \\
\hline 1. & Sort & $11(36.7 \%)$ & $12(40.0 \%)$ & $7(23.3 \%)$ & 5.40 & 2.37 \\
\hline 2. & Decimal & $12(40.0 \%)$ & $11(36.7 \%)$ & $7(23.3 \%)$ & 5.17 & 2.34 \\
\hline 3. & AutoSum & $12(40.0 \%)$ & $11(36.7 \%)$ & $7(23.3 \%)$ & 5.17 & 2.31 \\
\hline
\end{tabular}



Vol. 10, No. 11, 2020, E-ISSN: 2222-6990 ๑ 2020 HRMARS

\begin{tabular}{|c|c|c|c|c|c|c|}
\hline 4. & Wrap Text & $8(26.7 \%)$ & $15(50.0 \%)$ & $7(23.3 \%)$ & 5.57 & 2.31 \\
\hline 5. & Print Sheet & $5(16.7 \%)$ & $13(43.3 \%)$ & $12(40.0 \%)$ & 6.70 & 2.35 \\
\hline 6. & Insert Cells & $5(16.7 \%)$ & $13(43.3 \%)$ & $12(40.0 \%)$ & 6.57 & 2.30 \\
\hline 7. & Sort \& Filter & $8(26.7 \%)$ & $13(43.3 \%)$ & $9(30.0 \%)$ & 5.87 & 2.47 \\
\hline 8. & Insert Sheet & $7(23.3 \%)$ & $12(40.0 \%)$ & $11(36.7 \%)$ & 6.20 & 2.38 \\
\hline 9. & Change Font & $7(23.3 \%)$ & $9(30.0 \%)$ & $14(46.7 \%)$ & 6.53 & 2.70 \\
\hline 10. & $\begin{array}{l}\text { Insert } \\
\text { Function }\end{array}$ & $9(30.0 \%)$ & $12(40.0 \%)$ & $9(30.0 \%)$ & 5.80 & 2.55 \\
\hline 11. & $\begin{array}{l}\text { Merge } \\
\text { Center }\end{array}$ & 10(33.3\%) & $9(30.0 \%)$ & $11(36.7 \%)$ & 5.70 & 2.69 \\
\hline 12. & $\begin{array}{l}\text { Insert } \\
\text { Worksheet }\end{array}$ & 10(33.3\%) & $12(40.0 \%)$ & $8(26.7 \%)$ & 5.47 & 2.60 \\
\hline 13. & $\begin{array}{l}\text { Insert Sheet } \\
\text { Rows }\end{array}$ & $6(20.0 \%)$ & $12(40.0 \%)$ & $12(40.0 \%)$ & 6.23 & 2.47 \\
\hline 14. & $\begin{array}{l}\text { Change Font } \\
\text { Size }\end{array}$ & $5(16.7 \%)$ & $8(26.7 \%)$ & $17(56.7 \%)$ & 6.97 & 2.57 \\
\hline 15. & $\begin{array}{l}\text { Insert Sheet } \\
\text { Column }\end{array}$ & $6(20.0 \%)$ & $10(33.3 \%)$ & $14(46.7 \%)$ & 6.57 & 2.62 \\
\hline 16. & $\begin{array}{l}\text { Change } \\
\text { Format Cell }\end{array}$ & 10(33.3\%) & $9(30.0 \%)$ & $11(36.7 \%)$ & 5.60 & 2.79 \\
\hline Total & & $7(23.3 \%)$ & $14(46.7 \%)$ & $9(30.0 \%)$ & 5.97 & 2.11 \\
\hline
\end{tabular}

A total of 16 items was constructed to measure the level of proficiency using spreadsheet software (Excel) among students. Overall, students were found to e moderately skilled when $5(15.7 \%)$ to $12(40.0 \%)$ students did not master this skill. The mean score recorded for all items ranged from 5.17 to 6.97 with an overall mean score of 5.97. This situation illustrates that the students are not using this software as much during the study. The lowest mean scores recorded for this skill were for Decimal and AutoSum items. Meanwhile, the highest mean score is for the Change Font Size item.

TALE 6. Level of Proficiency in Using the Internet

\begin{tabular}{|c|c|c|c|c|c|c|}
\hline \multirow[t]{2}{*}{ No. } & \multirow[t]{2}{*}{ Items } & \multicolumn{3}{|c|}{ Mastery Level } & \multirow[b]{2}{*}{ Mean } & \multirow[b]{2}{*}{ SD } \\
\hline & & Unskilled & $\begin{array}{l}\text { Moderately } \\
\text { Skilled }\end{array}$ & Skilled & & \\
\hline 1. & e-SMP & 0 & $2(6.7 \%)$ & $28(93.3 \%)$ & 9.20 & 0.89 \\
\hline 2. & YouTue & 0 & $3(10.0 \%)$ & $27(90.0 \%)$ & 9.10 & 1.06 \\
\hline 3. & Putralast & 0 & $4(13.3 \%)$ & $26(86.7 \%)$ & 8.87 & 1.38 \\
\hline 4. & Google Maps & 0 & $8(26.7 \%)$ & $22(73.3 \%)$ & 8.37 & 1.67 \\
\hline 5. & Google Scholar & $2(6.7 \%)$ & $5(16.7 \%)$ & $23(76.7 \%)$ & 8.20 & 1.63 \\
\hline 6. & Watch video & $1(3.3 \%)$ & $6(20.0 \%)$ & $23(76.7 \%)$ & 8.67 & 1.47 \\
\hline 7. & Internet Explorer & $2(6.7 \%)$ & $5(16.7 \%)$ & $23(76.7 \%)$ & 8.50 & 1.63 \\
\hline
\end{tabular}




\begin{tabular}{lllllll} 
8. & Google Translate & $1(3.3 \%)$ & $5(16.7 \%)$ & $23(76.7 \%)$ & 8.73 & 1.55 \\
9. & Find information & 0 & $3(10.0 \%)$ & $27(90.0 \%)$ & 8.87 & 1.25 \\
10. & Online software & $1(3.3 \%)$ & $11(36.7 \%)$ & $18(60.0 \%)$ & 7.93 & 1.72 \\
11. & Generating log & $11(36.7 \%)$ & $7(23.3 \%)$ & $12(40.0 \%)$ & 5.83 & 2.82 \\
12. & Uploading material & $3(10.0 \%)$ & $7(23.3 \%)$ & $20(66.7 \%)$ & 8.07 & 1.84 \\
13. & Downloading material & $1(3.3 \%)$ & $7(23.3 \%)$ & $22(73.3 \%)$ & 8.30 & 1.56 \\
14. & uy \& sell online & $1(3.3 \%)$ & $10(33.3 \%)$ & $19(63.3 \%)$ & 7.87 & 1.87 \\
15. & Using email & $1(3.3 \%)$ & $5(16.7 \%)$ & $24(80.0 \%)$ & 8.60 & 1.63 \\
16. & Online games & $8(26.7 \%)$ & $8(26.7 \%)$ & $14(46.7 \%)$ & 6.37 & 2.75 \\
17. & Generating wesite & $9(30.0 \%)$ & $13(43.3 \%)$ & $8(26.7 \%)$ & 5.60 & 2.77 \\
18. & Read newspaper online & 0 & $12(40.0 \%)$ & $18(60.0 \%)$ & 7.77 & 1.59 \\
19 & Attach documents using & 0 & $8(26.7 \%)$ & $22(73.3 \%)$ & 8.37 & 1.61 \\
& email & & & & \\
20. & Sending emails using & $4(13.3 \%)$ & $\mathbf{9 ( 3 0 . 0 \% )}$ & $17(56.7 \%)$ & 7.30 & 2.28 \\
& Google Drive & & & & \\
\hline Total & $\mathbf{0}$ & $\mathbf{7 ( 2 3 . 3 \% )}$ & $\mathbf{2 3 ( 7 6 . 7 \% )}$ & $\mathbf{8 . 0 3}$ & $\mathbf{1 . 2 4}$ \\
\hline
\end{tabular}

A total of 20 items were constructed to measure the level of mastery of Internet skills among students. Overall, students were found to e proficient in using the Internet when they recorded a mean score of 8.03 with a standard deviation of 1.24 and no students were unskilled in using these skills. However, looking at each item, there are three items (producing logs and wesites as well as online games) that recorded a moderate mean score, when a total of $8(26.7 \%)$ to $11(36.7 \%)$ students were unskilled in this section. Meanwhile, students are most skilled in using e-SMP ( $M=9.20 ; \mathrm{SP}=0.89)$ and YouTue $(\mathrm{M}=9.10 ; \mathrm{SP}=1.06)$. This is ecause e-SMP is a system that must e used y students throughout the study, which is for course registration and checking the results from time to time throughout the lecture.

\section{Conclusion}

Overall, it can e concluded that most of the students studied were proficient in using word processing software (Word) when $83.3 \%$ of the students mastered it. This is followed $y$ Internet usage skills with $76.7 \%$, presentation software (PowerPoint) of $66.7 \%$, asic computer software of $36.7 \%$, and electronic spreadsheets (Excel) has of $30.0 \%$ of students mastering it. ased on these findings, it can e concluded that students master the Word processing software ecause they often used it to complete assignments throughout their studies. On the other hand, the second highest component, which is the internet software may e due to the Wi-Fi facilities provided $y$ the university or faculty to make it easier for students to find information. esides that, students or samples involved in this study also spend a lot of time surfing the Internet. Half of the students surveyed spending more than seven hours a week. The findings show that these students spend at least two to three hours a week surfing the Internet. All students were found surfing the Internet for email purposes. Internet facilities are also used for entertainment, curiosity, chat, file transfer, social networking, getting help and information, completing tasks and downloading software. Commonly, they used personal laptops and smartphones respectively.

Some students surf the Internet through smartphone applications only and some students use personal computer desktops. This is supported y the opinion of Siew et al. (2016) who said that although students are aware of the importance of technology in learning, students are more likely to use information technology and communication for social purposes rather 
than academics. ased on a study conducted y Gaarre et al. (2013), the use of moile phones or smartphones in most cases is less preferred than computer laptops due to the small screen size. However, for this study, students were found to e frequently using oth to surf the Internet.

The students were also found to own more than one computer. This proves that the students in this generation know the importance of technology and it can e said that computer ownership is a asic need for them. All students involved in this study owns a computer either a personal laptop or a personal desktop. When asked aout the source of their knowledge of computers, students were found to acquire the source of computer skills through more than one source. For example, through self-awareness and efforts, friends, the mass media and some attend courses to increase their knowledge (Jusoh et al., 2018). These students have high expectations that multimedia applications will e implemented in their classrooms (Sahrir \& Alias, 2011). Therefore, this survey can help the lecturers' design teaching and learning activities, also knowing the level of information and communication technology skills among students. In return, it can produce students who can compete in the jo market that requires current graduates to possess these generic skills.

\section{Contriution to Knowledge}

This study was conducted ased on the ASSURE model, which is to conduct an analysis of students' knowledge of information technology efore the lecture Multimedia in Language is implemented for a period of 14 weeks. This analysis of student knowledge is important to conduct to acquire students' existing knowledge and formulate the next learning process. This is ecause if the lecturer repeats the teaching that has een mastered y the students, it is likely that the teaching and learning sessions will e oring and students did not gain new knowledge. $y$ analyzing existing knowledge in the field of technology to students, lecturers can not only know the extent of students' existing knowledge, ut can also design more enjoyale teaching and learning activities. This analysis of technological knowledge is important to e carried out, especially among the Net generation who are well aware that the generation orn in the age of technology is growing rapidly. Therefore, of course this generation is no stranger to technological skills. However, the extent to which their technological knowledge needs to e identified to ensure that this technological knowledge is alanced for their social and learning needs. ased on the findings, the sample studied, namely the achelor of Arts Malay Language and Linguistics, who was orn around 1992 to 1995, the Net generation has fulfilled the information and communication technology skills. ased on the analysis, student is found to dominate asic computer skills such as word processing software and the Internet. However, they were found to e less proficient in presentation software (PowerPoint), asic computer software and electronic spreadsheets (Excel). Therefore, ased on this analysis, the lecturer will focus on learning three skills that have not een mastered y students. For example, to achieve their learning outcomes create a language project using multimedia software, students are exposed to the PowerPoint function in producing animation. For that, students need to explore and utilize PowerPoint software to produce multimedia projects in groups.

\section{Implication of the Findings}

This study of students' knowledge analysis of technological knowledge not only helps course lecturers to design teaching and learning methods that are appropriate to the needs of students, ut also provides an initial overview of technological knowledge among Net generation students. Although they were orn in the age of technology is growing rapidly and 
spending a lot of time with technology, ut the knowledge and skills possessed y these students can still e categorized as low. This is ecause most of the courses conducted apply technological elements either in terms of lecture delivery or the need for students to produce assignments and present the results of their assignments.

ased on the findings of this study, there is still a lot of room for improvement to improve the knowledge and skills of these students. This is ecause of the need and intense competition in the world of work. The need for technological skills is among the skills demanded y current employers. Therefore, to enale Malay language students is ale to compete with students in other areas, changes in teaching and learning activities are needed.

\section{Author Information}

Dr. Zuraini Jusoh is a senior lecturer in the Department of Malay Language, Faculty of Modern Language and Communication, Universiti Putra Malaysia (UPM). Her expertise is teaching Malay as a First Language.

\section{References}

Madar, A. R., Ad Aziz, M. A., Ad. Razzaq, A. R., Mustafa, M. Z. \& untat, Y. (2008), Kemahiran Employaility agi Memenuhi Keperluan Industri. Retrived from https://www.google.com/search?q=Ahmad+Rizal+Madar+\%2+Kemahiran+Employaili ty\&rlz=1C1CHZL_enMY749MY749\&oq=Ahmad\&aqs=chrome.1.69i57j69i59/2j0l3.383 9j0j8\&sourceid=chrome\&ie=UTF-8.

Agus, P., Ratnawati, N. \& Nevy, F. A. (2016). Pengemangan Pemelajaran lended Learning Pada Generasi Z. Jurnal Teori dan Praksis Pemelajaran IPS, 1(1), 70-77.

Aladdin, A., Hamat, A. \& Yusof, M. S. (2004). Penggunaan PK (Pemelajaran ahasa erantukan Komputer) dalam Pengajaran dan Pemelajaran ahasa Ara seagai ahasa Asing: Satu Tinjauan Awal. GEMA Online ${ }^{T M}$ Journal of Language Studies, 4(1), 129-145.

Auzar, N. (2012). Keerkesanan Penggunaan Perisian Asas Memaca. GEMA Online ${ }^{T M}$ Journal of Language Studies, 12(2), 629-644.

ers, M. U. (2010). Issue Editor's Notes. Retrived from https://onlinelirary.wiley.com/doi/pdf/10.1002/yd.369.

Davis, P. (1997). What Computer Skills Do Employee Expect From Recent College Graduates. The Journal, 25(2), 74-79.

Gaarre, S., Gaarre, C., Din, R., Mohd Shah, P. \& Adul, A. (2013). Using Moile Faceook as an LMS: Exploring Impeding Factors. GEMA Online ${ }^{T M}$ Journal of Language Studies, 13(3), 99-115.

Guilford, J. P. (1956). Fundamental statistics in psychology and education, McGraw-Hill ook Company: New York.

Mohd, A. H., Salim, J., Adullah, S., Judi, H. M. \& Mat Zin, N. A. (2001). Computer Competency Level ased on Jo Function in Malaysia. Asia-Pacific Journal of Information Technology and Multimedia, 1, 40-57.

Mohamed, H., Judi, H. M., Noor, M. S. F. \& Yusof, Z. M. (2012). Jurang Digital dan Pendidikan di Luar andar: Tahap Literasi Teknologi Maklumat dan Komunikasi Pelajar. Jurnal Teknologi Maklumat dan Multimedia Asia-Pasifik, 1(2), 39-45.

Kiam-Sam, H. \& Aziz, A. N. (2014). Technology Use and Digital Learning Characteristics Among Malaysian Undergraduates, Sains Humanika, 2(1), 117-124. 
Alias, N. (2004). Taksiran Keperluan Pengurusan teknologi Maklumat di Kalangan Guru Sains Dalam Perkhidmatan. Jurnal Penyelidikan MPL, 5(87)-102.

Emi, M. A. (2010) (ed.). Rumusan Amalan, Tend dan Caaran Pelaksanaan e-Pemelajaran di IT Malaysia. Kuala Lumpur: Jaatan Pengajian Tinggi, Kementerian Pengajian Tinggi Malaysia.

Pitchan, M. A., Omar, S. Z., olong, J. \& Ahmad, A. H. (2017). Analisis Keselamatan Sier dari Persepktif Persekitaran Sosial: Kajian Terhadap Pengguna Internet di Lemah Klang. $e$ angi, 12(2), 16-29.

Ismail, M. H. (2012). Kajian Mengenai Keolehpasaran Siswazah di Malaysia: Tinjauan dari Perspektif Majikan. Prosiding Persidangan Keangsaan Ekonomi Malaysia (PERKIM), VII (2), 906-913.

Sahrir, M. S. \& Alias, N. A. (2011). A Study on Malaysian Language Learners Perception Towards Learning Araic via Online Games. GEMA Online ${ }^{T M}$ Journal of Language Studies, 11(3), 129-145.

Omar, N. H., Mohamad, M. A. \& Razak, N. A. (2006). Penilaian Kecekapan Teknologi Komunikasi Maklumat (TMK) dalam kalangan Pelajar Sains Sosial. Jurnal Pendidikan Universiti Malaya, 26, 63-77.

Olinger, D., \& Olinger, J. (2005). Is It Age or IT: First Steps Toward Understanding the Net Generation. Retrived

from https://www.google.com/search?q=Diana+Olinger+\%2+lt+is+Age+or+IT\&rlz=1C1CHZ L_enMY749MY749\&oq=Diana+Olinger+\%2+It+is+Age+or+IT\&aqs=chrome..69i57.184 36j0j8\&sourceid=chrome\&ie=UTF-8.

Pallant, J. (2001). SPSS survival manual: A step y step guide to data analysis using SPSS for Windows (Version 12), Open University Press: New York.

Siew, M. T., Kean, W. L, Puvaneswary, M., Mohd Jaafar, N., Choon, K. T. \& Ahmad, N. I. (2016). ICT Tools Patterns of Use Among Malaysia ESL Undergraduates. GEMA Online ${ }^{T M}$ Journal of Language Studies, 16(1), 49-65.

Mustafa, S. E.. \& Hamzah, A. (2011). Media aharu yang aharu: Tend Penggunaan Jaringan Sosial dalam Kalangan Pengguna di Malaysia. Jurnal Pengajian Media Malaysia, 13(2), 93-110.

Suthagar, N., Md. Yunus, M. R., \& Ahmad, A. (2011). Net Generation Student Teachers: How Tech-Savvy Are They? Asia Pacific Journal of Educators and Education, 26(1), 71-89.

Mahamod, Z., \& Mohamad, N. A. (2011). Persepsi Guru Tentang Penggunaan Aplikasi Multimedia dalam Pengajaran Komponen Sastera ahasa Melayu. GEMA Online ${ }^{T M}$ Journal of Language Studies, 11(3), 163-177.

Jusoh, Z., Said, R., \& Kiram, M. N. (2018). Kemahiran Perisian Pemprosesan Perkataan Dalam Kalangan Pelajar idang ahasa Melayu. Paper presented at PASAK3 of the International Conference Social Science and Humanities, Kolej Universiti Antaraangsa Selangor, 2324 April.

Jusoh, Z. (2018). Pengetahuan Teknologi Maklumat dalam Kalangan Pelajar idang ahasa Melayu. Paper presented at SALPM10 of the International Conference Linguistic and Malay Language Cultivation, 6-7 Feruary. 